\title{
TASAS DE RETORNO DE LA EDUCACIÓN SUPERIOR EN HONDURAS
}

Nelson Raudales García ${ }^{1}$

Cesar H. Ortega Jiménez ${ }^{2}$

\section{Resumen}

En el presente artículo se estima estadísticamente las tasas de retorno de la educación en Honduras. Considerando los diversos niveles de la misma, se describe la ecuación de regresión múltiple utilizada para tal fin, sin dejar de considerar otras estimaciones realizadas por otros autores. Los resultados han sido calculados en base a las fuentes de las diversas Encuestas de Hogares de Propósitos Múltiples (EPHPM) realizada por el Instituto Nacional de Estadísticas de Honduras (INE) para los años respectivos de estudio y análisis. Las bases de datos puestas a disposición, son de fácil uso para tratamiento y manipulación por medio del programa para manejo estadístico SPSS, almacenados en micro datos. Los principales resultados encontrados reflejan hallazgos importantes, a saber: uno de ellos principalmente es el nivel y/o grado de asociación entre la variable educativa para sus diversos niveles y los ingresos monetarios de las personas y, que los resultados de las estimaciones de las tasas de retorno del sistema educativo muestran que las mismas son mayores para el nivel de educación secundaria en comparación con las tasas de educación primaria y el nivel de educación superior. Ello pudiera verse reflejado en los sistemas de cuentas nacionales por ejemplo en las tasas de participación de la población con los niveles académicos mencionados en las diversas actividades económicas en los diversos sectores de la economía, además comparativamente éstos reflejan mayores aportaciones económicas al país, reflejado por medio del Producto Nacional Bruto.

\footnotetext{
${ }^{1}$ Profesor del Instituto de Investigaciones Económicas y Sociales (IIES-UNAH).

2 Director del Instituto de Investigaciones Económicas y Sociales (IIES-UNAH).
} 


\begin{abstract}
In this article it is estimated statistically rates of return to education in Honduras considering various levels of it, describes the equation multiple regression used for this purpose, while consider other estimates made by other authors.
\end{abstract}

The results of this evaluation have been calculated based onvarious sources hosuehold surveys for Multiple Purposes (ephpm) conducted by the National Statistics Institute Honduras (INE) for the respective years of study and analysis, these databases are made available to easy to use for treatment and handling through SPSS management program stored in micro data.

The main results reflect important results, namely one is primarily the level and / or degree of association between education variable for various levels and monetary income people and that the results estimates of rates of return of the system education show the same level are higher for primary education compared to rates secondary education and higher education level. This could be reflected in accounting systems eg national participation rates population with more academic standards in various economic activities in various sectors of the economy, and they reflect comparatively greater financial contributions to the country, reflected by half of GNP.

\title{
Introducción
}

Los resultados que la educación genera sobre el desarrollo económico, tradicionalmente han sido medidos a través del método de la tasa interna de retorno de la educación. Sin embargo, dicho método demanda la utilización del supuesto de que los ingresos salariales se igualan a las productividades y que las diferentes categorías de trabajo, clasificadas de acuerdo a los años de escolaridad de los trabajadores, se establecen en sustitutos en la producción. Además de ello, el método supone perfecta movilidad social de la mano de obra y la existencia de competencia perfecta en los mercados de trabajo. 
El cálculo de la tasa interna de retorno de la educación para los trabajadores perceptores de ingresos, constituye una manera de medir los aspectos relacionados al salario y sus efectos en la decisión de educarse y de acumular capital humano. De esta forma, si se considera una visión de mediano y largo plazo sobre el crecimiento y desarrollo económico, es decisiva la contribución que puede ejercer la variable educación. De ahí la necesidad de suscitar el mejoramiento de la formación y adiestramiento del capital humano, para fomentar la capacidad productiva a mediano y largo plazo, la prosperidad de los países.

Los modelos desplegados en tal dirección, intentan definir los determinantes de los salarios y terminan por estimar la incidencia sobre los salarios, de las variables ligadas a la educación y la acumulación de aprendizaje, como lo son los años de escolaridad y la posible experiencia laboral potencial. En el trabajo precursor de Mincer (1974), se establece la metodología del cálculo de la tasa de retorno de la educación, mediante la estimación de funciones de ingreso laboral. Mincer encuentra parámetros de validez empírica de la existencia de una relación de causalidad positiva, que se refleja en los niveles educativos a incrementos o mejoras en los ingresos laborales, lo cual es consistente con la tradicional teoría del capital humano.

Uno de los hallazgos importantes encontrados en el presente, tiene que ver con la evidencia de la tendencia significativa y diferenciada de las tasas de rendimiento para los años de análisis, es decir, como resultado de los aumentos educativos la tasa de retorno interna de la educación varía entre cada uno de los niveles.

A través de la estimación econométrica de ecuaciones salariales, se puede observar los valores de la tasa interna de retorno de la educación y de los beneficios que la educación brinda para los diversos asalariados con diferentes años de escolaridad. Adicionalmente, los resultados econométricos concuerdan con la evidencia de los salarios relativos, que muestran un detrimento de las personas con menos niveles educativos frente a los más educados, es decir con niveles universitarios de educación. 
El propósito del análisis del retorno a la inversión en educación que se realiza en el presente trabajo se pudiera abordar desde una perspectiva de competitividad, atendiendo a la importante incidencia de los progresos en formación, práctica y asimilación de disciplina de operación, sobre los resultados productivos del factor trabajo.

Así pues en los contenidos posteriores, en el epígrafe (2) se describen algunos hechos encontrados en cuanto a ingresos y niveles educativos, en el epígrafe (3) se describen y analiza la relación nivel educativo e ingresos; en el epígrafe (4) se realiza una breve descripción relacionada a la teoría del capital humano y las tasas de retorno, en el epígrafe (5) se describe el modelo econométrico para tal fin; en el epígrafe (6) se describen las fuentes de datos a utilizar que se utilizarán; en el epígrafe (7) se describe los resultados y el análisis de la estimación; en el epígrafe (8) se describen ciertas consideraciones generales del análisis; en el epígrafe (9) se menciona la principal bibliografía utilizada para tal fin.

\section{2.- ALGUNOS HECHOS ENCONTRADOS EN CUANTO A INGRESOS Y NIVELES} EDUCATIVOS

\section{Niveles de escolaridad de la población en Honduras.}

Honduras, puede ser considerado un país de bajo índice de desarrollo humano, ello debido a que posee niveles aún bajos en cuanto a condiciones de vida (ingresos bajos), baja escolaridad (promedio 6 años de educación) y a nivel de salud se refiere.

Al considerar la educación como elemento para el mejoramiento de las condiciones de vida, es plausible estimar que dicho indicador es considerado bajo si se quisiera alcanzar niveles decorosos de vida, además considerar que dicha limitante en cuanto a la escolaridad de la población se refiere, difícilmente se logrará salir de condiciones paupérrimas que ponen en detrimento el nivel de salud de las personas; al considerar la ubicación geográfica es aún más diferenciada dicho indicador, en donde por ejemplo mientras en las zonas urbanas el promedio de educación para el sexo masculino es de 7.1 años, en la misma zona para las mujeres es de 6.6. Cuanod se describe dicho 
indicador para el área rural es más dramática la situación, en donde puede reflejarse que para dicha zona el promedio educativo para los hombres es 3.0 y para la mujer 2.4 (Cuadro No.1).

\section{Cuadro No. 1: Escolaridad promedio de la población de 15 años y más por sexo.}

\begin{tabular}{|c|c|c|c|}
\hline \multirow[t]{2}{*}{ AREA } & \multicolumn{2}{|c|}{ SEXO } & \multirow[t]{2}{*}{ TOTAL } \\
\hline & MASCULINO & FEMENINO & \\
\hline URBANA & 7.1 & 6.6 & 6.9 \\
\hline RURAL & 3.0 & 2.4 & 2.8 \\
\hline TOTAL & 4.7 & 4.8 & 4.7 \\
\hline
\end{tabular}

Fuente: Elaboración propia en base a INE, Ephpm, 2005.

Además, como elemento vinculante al proceso de analizar la evolución de los salarios medios del país, es importante revisar algunos hechos estilizados sobre la relación entre las variables de ingresos y educación, mismas que juegan un rol importante en la estimación de los indicadores comentados previamente.

Dado que no se dispone de los ingresos monetarios por departamentos, como una variable de aproximación a los mismos, se ha procedido a utilizar el PIB per cápita. Aunque la correlación no necesariamente pueda implicar alguna causalidad, sí puede dar una idea con respecto al grado de asociación estadístico que existe entre el PIB per cápita y los años de escolaridad. En el cuadro 2 se puede observar la correlación positiva existente entre PIB per cápita y años de escolaridad (reflejado por medio de los niveles educativos de los trabajadores). Se destaca el incremento del coeficiente de correlación para las dos variables en los años descritos. La interpretación de los resultados debe manejarse con mucho cuidado, pues dicho coeficiente no necesariamente pudiera reflejar aspectos que posiblemente requieran mayor nivel de análisis y estudio que la causalidad existente entre las dos variables descritas, es decir que las mejoras en el PIB per cápita son el resultado de los mayores niveles educativos. Para determinar los rendimientos educativos, se debe realizar la estimación de 
ecuaciones salariales, las cuales nos mostrarán los retornos o beneficios respecto a la inversión en educación en cada uno de sus niveles.

\section{Cuadro 2: Relación Ingreso Vs. Educación (en sus diversos niveles)}

\begin{tabular}{|lcc|}
\hline \multicolumn{1}{|c|}{ Correlación } & 1999 & 2005 \\
\hline Pib- Edu Primaria & 19.0 & 22.0 \\
PIB-Edu. secundaria & 31.0 & 38.0 \\
PIB-Edu. Superior & 37.0 & 45.0 \\
\hline
\end{tabular}

Fuente: Elaboración propia en base a EPHPM, 1999, 2005.

Además, si se desease visualizar aún mejor la realización, ésta pudiera observarse a través del Gráfico No. 1 en el cual se visualiza el comportamiento del PIB per cápita y los años de escolaridad (niveles de educación) para los años previamente mencionados.

\section{Grafico No.1 Relacion o asociación entre} Ingreso-Nivel educativo

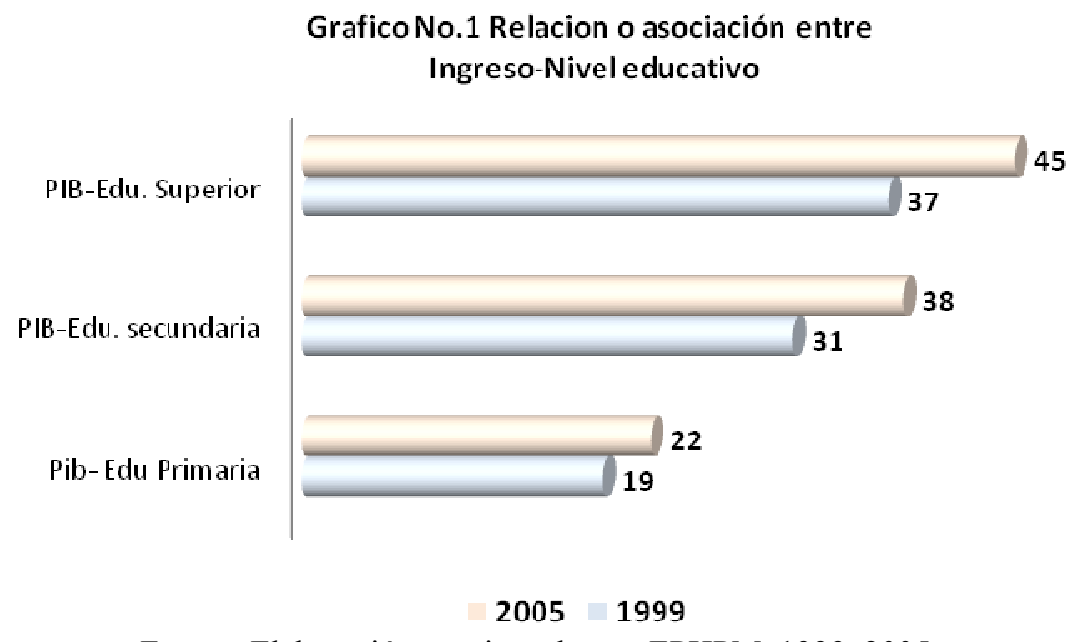

Fuente: Elaboración propia en base a EPHPM, 1999, 2005.

\section{3.- Relación nivel educativo e ingresos}

Además del análisis respectivo de relación o asociación (correlación) en cuanto a medición ingresos-educación, es posible vislumbrar otra comprobación en cuanto a los ingresos. Es por ello que en la gráfica 2 se analiza entonces la relación antes mencionada, observando que para el año 2005, las personas con mayor nivel educativo logran obtener ingresos considerables, no así las personas con menores niveles, ya que éstos lograrán acumular ingresos inferiores a las primeras. Es el caso por ejemplo de los 
que permanecen sin nivel, que logran alcanzar apenas la mitad de lo que ganan las personas con educación media, representado en este caso por personas con nivel educativo de secundaria.

Gráfico No. 2: Relación nivel educativo Vs ingreso percápita de las personas, 2005.

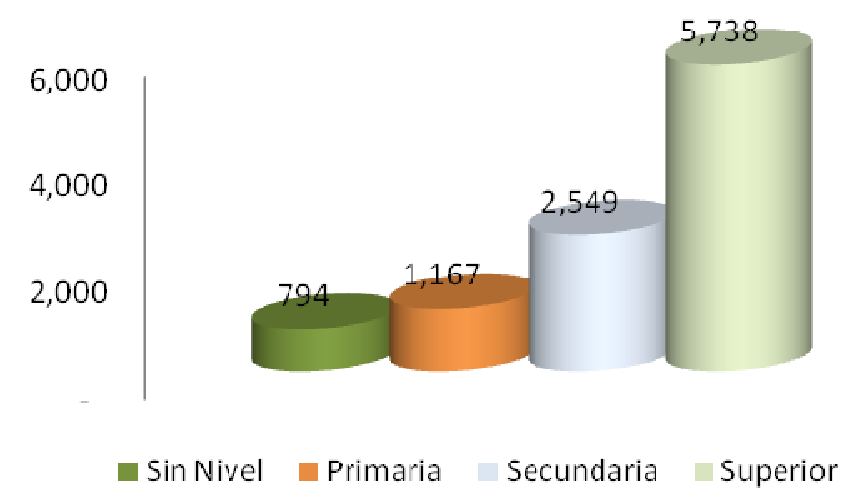

Fuente: Elaboración propia en base a EPHPM, 2005.

\section{4.- LA TEORIA DEL CAPITAL HUMANO Y LA TASA DE RETORNO}

Diversos estudios realizados en torno a los determinantes del crecimiento económico y de la productividad de la economía, han mostrado que las inversiones efectivas en capital humano contribuyen de manera significativa con una aportación importante, complementando las contribuciones provenientes de la inversión en capital fijo.

El rendimiento de dichas inversiones en capital humano se puede pensar desde el punto de vista individual, en el cual existe un sacrificio de rentas presentes con el objeto de aumentar los ingresos futuros, aportando a la actividad productiva un trabajo más calificado y con mayor productividad.

La teoría del capital humano a nivel empírico, hasta hace pocas décadas era algo aún incipiente, hoy en día, dicha teoría es bastante más amplia. En el presente trabajo, se inicia de la versión de estudios que apoyan por el supuesto que existe una relación directa entre los niveles de educación de los individuos y sus niveles de productividad. En este sentido, individuos con niveles altos de preparación (en mayor parte preparación académica) y por ende con mayor productividad devengarán ingresos más altos. 
Por otra parte, las decisiones acertadas de invertir en educación están determinadas a nivel económico por análisis y relación de tipo costo-beneficio. Los beneficios de la educación están representados por el valor presente del incremento del ingreso asociado al acceso a niveles más elevados de educación. Al mismo tiempo, los costos de la decisión están dados por el ingreso sacrificado durante los años de asistencia, sumados a los gastos en las matrículas y los gastos asociados para tal fin. El papel que puede jugar el gobierno, desde un punto de vista social, es importante. De esta manera, una asignación eficiente de recursos en el sector educativo puede desencadenar efectos globales que se manifiestan en mejoras en la productividad global y mejoramiento de la economía y en la formación de procesos integrales de progreso social que incidirá en el mejoramiento de la vida de las personas.

Los estudios empíricos que intentan abordar la problemática del capital humano, parten de la base de que la productividad y la remuneración al trabajo y que dependen en esencia de las inversiones tanto públicas como privadas en conocimiento (en aspectos como los relacionados a aprender haciendo) e información.

No obstante, no se deben desechar puntos de vista como el de la economía de la salud, que también produce una gran incidencia en los procesos de acumulación de capital humano, reflejado por ejemplo en educación, la cual de una u otra forma formará o capacitará a la población con el objeto de mejorar y prevenir situaciones que puedan poner en riesgo la salud de las personas del núcleo familiar o de una comunidad.

Con dichos planteamientos, a continuación se describe el modelo econométrico, que permitirá cuantificar la tasa interna de retorno de la educación y la incidencia que tiene sobre las mejoras salariales, la acumulación de capital humano a través de mayores niveles educativos. 


\section{Gráfico No. 3: Perfil de Salarios por nivel educativo y edades}

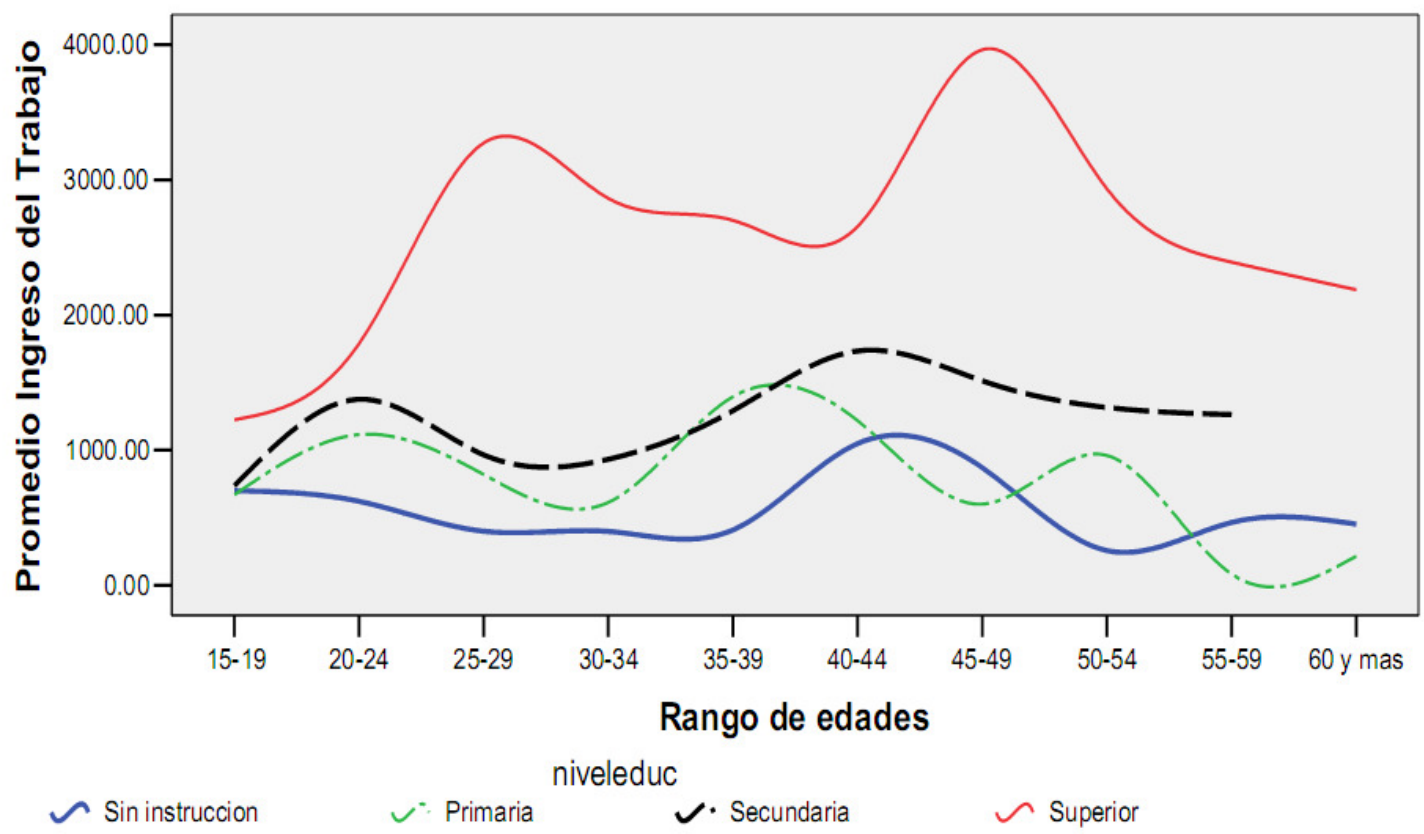

Fuente: Elaboración propia en base a EPHPM, 2005.

\section{5.- EL MODELO ECONOMÉTRICO}

El modelo a utilizar se designa modelo simple o función minceriana, el cual consiste en una función del ingreso, en el que se relaciona el logaritmo del salario en función de los años de escolaridad de las personas, la experiencia potencial, y el término cuadrático de la experiencia potencial, es decir descrito de la siguiente forma:

$$
\log \left(W_{i}\right)=\beta_{0}+\beta_{1} * \operatorname{Edu}_{2}+\beta_{2} * \operatorname{Expi}+\beta_{3} * \operatorname{Exp}_{\mathrm{i}}+\varepsilon
$$

La ecuación anterior puede interpretarse como la tasa de retorno de la educación, el coeficiente que acompaña los años de educación reflejados en la $\beta_{1}$.

Posteriormente al modelo simple en la ecuación de Mincer, se hace pertinente el modelo de Spline, el cual se caracteriza con el anterior ya que éste permite recoger los retornos de la tasas de educación en los niveles de primaria, secundaria y superior tal como se ha previsto en el presente, de igual forma la ecuación que capturaría dichos coeficientes está dada por la ecuación (2). 
$\log (w)=\beta+\beta * \operatorname{Edu}+\beta * \operatorname{Exp} .+\beta * \operatorname{Exp}_{i}^{2}+\beta_{4} * D_{s}\left(\operatorname{Edu}_{i}-11\right)+\beta_{5} * D_{u} *\left(\operatorname{Edu}_{i}>11+e\right.$

Donde $D_{s}$ es una variable binaria (Dummy) que toma el valor de 1 si la persona ha alcanzado secundaria completa o ha completado 12 años de educación; y toma el valor de cero en los demás casos. Así mismo, la variable $\mathrm{D}_{\mathrm{U}}$ toma el valor de 1 cuando la persona posee más de 12 años de educación, y en el resto de casos cero.

Es importante mencionar, que en este trabajo se utiliza y hace referencia a la ecuación del trabajo de Tenjo (1993). Según la misma, la tasa de retorno por un año adicional de educación primaria es $\beta_{1}$, la de un año adicional de educación en secundaria es la suma de los índices de $\beta_{1}+\beta_{4} y$ la de un año adicional de educación universitaria está representada por la suma de los parámetros $\beta_{1}+\beta_{5}$. El indicador $\beta_{4}$ representa un 'premio' a la educación secundaria, mientras que el coeficiente $\beta_{5}$ es el premio a la educación universitaria. De esta forma, si el producto de los dos parámetros resulta positivo en la estimación realizada, indica que la tasa de retorno de la educación secund universitaria) es mayor que la de primaria. En la gráfica 3, se puede apreciar el anterior análisis que se deriva de la ecuación Spline.

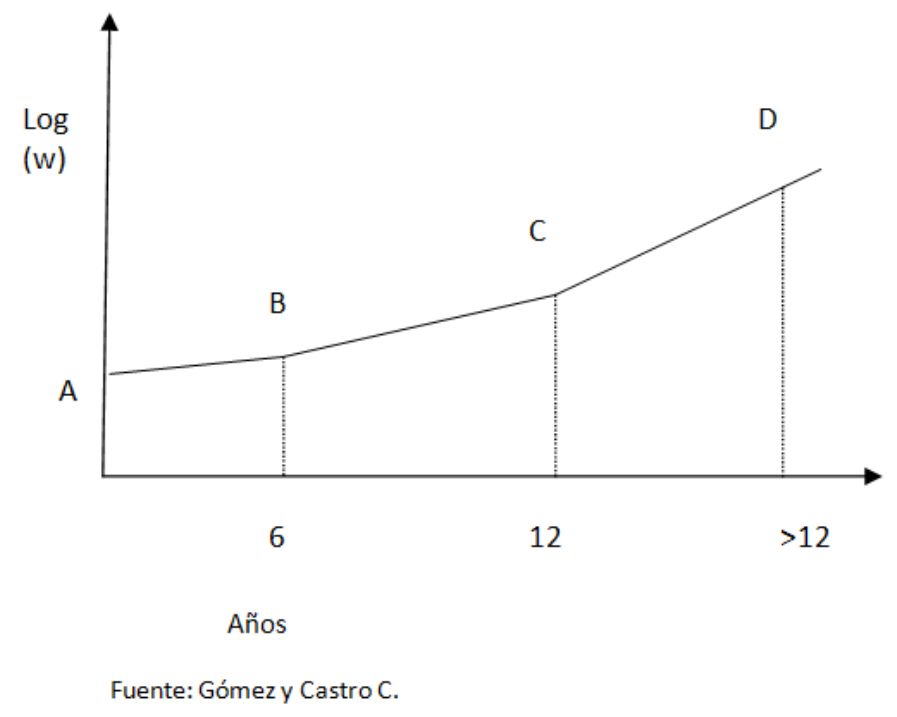


La ecuación en mención está formada por varios interceptos en su recta, los cuales están representados por las letras $\mathrm{ABCD}$, en las que se describe los diferentes niveles educativos: es el caso para el segmento $\mathrm{AB}$ representa el nivel de educación primaria (misma que corresponde al periodo entre 0 a 6 años de educación), el segmento BC representa la educación secundaria (el cual acumula o refleja en dicho segmento 12 años de educación), y por último en el segmento CD el cual está representando el nivel de la educación universitaria (la cual acumula más de 12 años de educación). El rasgo principal de dicha ecuación es que a medida se incrementa el nivel educativo se reflejarán mejores salarios para los individuos.

\section{6.- Fuentes de datos y años seleccionados.}

La principal fuente de datos para el establecimiento y generación de los indicadores relacionados a las tasas en mención provienen de las encuestas de Hogares de Propósitos Múltiples (EPHPM) que realiza el INE, así mismo es importante mencionar que con dichos datos se puede calcular dichas tasas para periodos longitudinales o bien realizarlo de manera transversal, tal como se está analizando en el presente artículo. Además es pertinente recalcar que las tasas en mención pueden calcularse para los niveles de educación primaria, secundaria y educación superior, tal como se plantea en el presente en donde se está analizando dos periodos de corte transversal (análisis para los años 1999 y 2005).

\section{7.- Resultados y análisis de la Estimación.}

Para efectos del análisis pertinente de los resultados que se generarían en el presente, la ecuación (2) se estimó utilizando el Método de Mínimos Cuadrados Ordinarios (MCO).

\section{Cuadro No. 3: Tasas internas de retorno según nivel educativo}

\begin{tabular}{|c|c|}
\hline Nivel educativo & TIR \\
\hline Primaria & 13.3 \\
\hline secundaria & 22.5 \\
\hline Superior & 15.7 \\
\hline
\end{tabular}




\section{8.- Consideraciones generales.}

Los resultados de las estimaciones econométricas ofrecen evidencia del estado de los avances en los años de educación, lo cual da una idea de la tendencia que en promedio se observó para la población en estudio en los instrumentos secundarios como ser las EPHM.

El modelo presentado reconoce la importancia de las habilidades productivas derivadas de la elevación de los niveles de educación de los individuos. Lo anterior, se manifiesta en los valores estimados de los coeficientes que capturan los premios a la educación, los cuales resultaron estadísticamente significativos y diferentes de cero; y con los signos esperados. Deduce también que la acumulación de capital humano tiene un efecto importante sobre la productividad y a partir de allí sobre los niveles de ingreso y bienestar de los individuos como de sus familias.

Como tendencia general, existe evidencia empírica de que las mejoras salariales han estado afectadas positiva y significativamente por la variable educación, y que estas mejoras de los salarios en el mercado laboral representa diferencias significativas tanto a nivel urbano como a nivel de género. Los hombres superan los ingresos de las mujeres. De acuerdo a la evidencia empírica reportada en este trabajo, se podría afirmar que cuando los accesos a la educación se difunden y los niveles educativos se elevan, la oferta en los mercados laborales se va cualificando y la tasa de retorno podría tender a decrecer, ello al no considerar la demanda posible de profesionales que pudiera absorber el mercado laboral.

Desde este punto de vista, se debe considerar que la adquisición de conocimientos a través de la educación es un proceso dinámico, pues inversiones tempranas en educación habilitan posteriores inversiones en formación de capital humano.

De esta forma, la educación podría contribuir a eliminar las desigualdades en la distribución del ingreso. Además, puede indicar el papel fundamental que tiene la 
educación en eliminar o disminuir la pobreza absoluta, así como el mejoramiento a nivel general de las condiciones de vida de la población. 


\section{Bibliografía.}

- Bonnefoy, et, al (2006) Capital humano en la región de Tarapacá. Universidad Adolfo Ibañez. Chile

- Brynin, M. and Longhi, S. (2006) The wage effects of graduate competition. Colchester: Institute for Social and Economic Research, University of Essex. GLM292.

- Conlon, G. and Chevalier, A. (2003) Financial returns to undergraduates and tuition fees. Council for Industry and Higher Education. GLM120.

- Council for Industry and Higher Education(2003) The value of higher education. London, UK: Council for Industry and Higher Education. ISBN 1874223-42-4 9781874223429 GLM200.

- Cuevas, Homero. 1996. "El Capital Humano en el Sistema de Precios", Cuadernos de Economía 15, 24, Bogotá.

- Greene, William H. 1998. Análisis Econométrico, Prentice Hall, tercera edición.

- Institute of Education (2001) The wider benefits of higher education. Bristol, UK: Higher Education Funding Council for England/Smith Institute. GLM78.

- Machin, S. and McNally, S. (2007) Tertiary education systems and labour markets. Paris: Organisationfor Economic Co-operation and Development. GLM290.

- Mincer, Jacob. 1974. "Schooling, Experience and Earnings", Studies in Human Behavior and Social Institutions .

- PriceWaterhouseCoopers (2007) The economic benefits of a degree. London: Universities UK. ISBN 1-84036-14849781840361483 GLM295.

- Tenjo, Jaime. 1993. "Evolución de los retornos a la inversión en educación 1976-1989”. Revista Planeación y Desarrollo 24, edición especial, diciembre, Bogotá, DNP. 